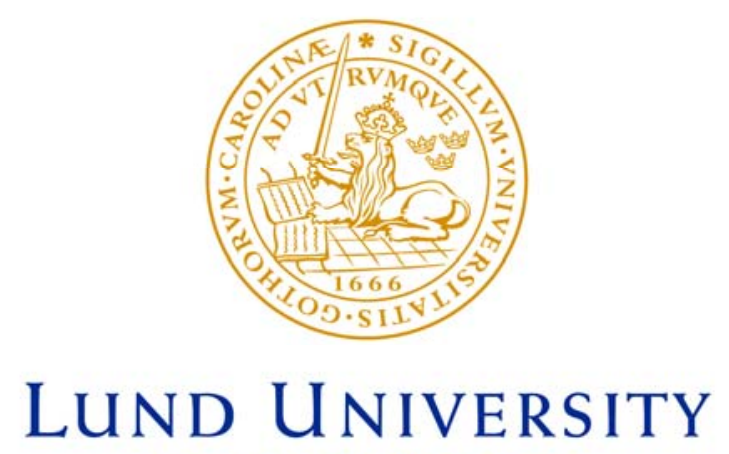

Faculty of Medicine

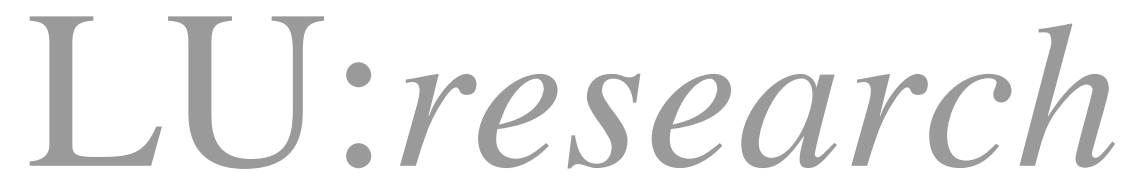

Institutional Repository of Lund University

This is an author produced version of a paper published in Blood. This paper has been peer-reviewed but does not include the final publisher proof-corrections or journal pagination.

Citation for the published paper:

Johansson, Maria K and de Vries, Teun J and Schoenmaker, Ton and Ehinger, Mats and Brun, Ann Cm and Fasth, Anders and Karlsson, Stefan and Everts, Vincent and Richter, Johan.

"Hematopoietic stem cell targeted neonatal gene therapy reverses lethally progressive osteopetrosis in oc/oc mice" Blood, 2007, Issue: Mar 1.

Access to the published version may require journal subscription.

Published with permission from: American Society of Hematology 


\section{Hematopoietic stem cell targeted neonatal gene therapy reverses lethally progressive osteopetrosis in oc/oc mice}

Maria K Johansson, Teun J. de Vries, Ton Schoenmaker, Mats Ehinger, Ann CM Brun, Anders Fasth, Stefan Karlsson, Vincent Everts and Johan Richter

From the Department of Molecular Medicine and Gene Therapy, Lund University, Lund, Sweden, Department of Periodontology and Oral Cell Biology, Academic Center for Dentistry Amsterdam (ACTA), Universiteit van Amsterdam and Vrije Universiteit, Amsterdam, The Netherlands, Department of Pathology, Lund University, Lund, Sweden and the Department of Pediatrics, Göteborg University Gothenburg, Sweden

Supported by the Swedish Children's Cancer Foundation, Lund University Hospital Foundations and Clinical Research Support (ALF) from Lund University Hospital.

Corresponding author: Dr Johan Richter, M.D., Ph.D., Molecular Medicine and Gene Therapy, Lund University, 22184 Lund, Sweden, Telephone: +46 4622205 87, Fax: +46 4622205 68, E-mail: Johan.Richter@med.lu.se

Word count: 5380

Abstract word count: 200

Scientific category: GENE THERAPY

Running head left: JOHANSSON et al

Running head right: GENE THERAPY OF OSTEOPETROSIS IN OC/OC MICE 


\begin{abstract}
Infantile malignant osteopetrosis (IMO) is a fatal disease caused by lack of functional osteoclasts and the only available treatment is hematopoietic stem cell (HSC) transplantation. In the majority of patients the TCIRG1 gene, coding for a subunit of a proton pump essential for bone resorption, is mutated. Oc/oc mice have a deletion in the homologue gene (tcigrl) and die at 3-4 weeks, but can be rescued by neonatal transplantation of HSCs. Here HSC targeted gene therapy of osteopetrosis in the oc/oc mouse model was developed. Oc/oc fetal liver cells depleted of Ter119-expressing erythroid cells were transduced with a retroviral vector expressing tcirgl and GFP, and subsequently transplanted i.p. to irradiated neonatal oc/oc mice. 8/15 mice survived past the normal lifespan of oc/oc mice. In vitro osteoclastogenesis revealed formation of GFP positive osteoclasts and bone resorption, albeit at a lower level than from wild-type cells. The skeletal phenotype was analyzed by X-ray and histopathology and showed partial correction at 8 weeks and almost normalization after 18 weeks. In summary, osteopetrosis in oc/oc mice can be reversed by neonatal transplantation of gene modified HSCs leading to long-term survival. This represents a significant step towards the development of gene therapy for osteopetrosis.
\end{abstract}




\section{Introduction}

The osteoclast and the osteoblast are two important mediators in the tightly regulated process of bone remodeling. The bone-forming osteoblasts are of mesenchymal origin while the multinucleated osteoclasts that are responsible for bone resorption, are derived from hematopoietic stem cells (HSCs). The balance between production and destruction of bone can be disturbed, and this will result in either reduction (osteoporosis) or increase (osteopetrosis) of bone mass.

Osteopetrosis comprises a heterogeneous group of diseases with varying degree of osteoclast dysfunction and the most severe form is called infantile malignant osteopetrosis (IMO) ${ }^{1}$. Children with this rare disorder have normal or elevated numbers of osteoclasts, which are unable to resorb bone due to defects in the acidification of the subcellular space between the osteoclast and the bone surface ${ }^{2-5}$. Other cellular processes, like cytoskeletal organization and ruffled border membrane formation, have also been suggested to be disturbed ${ }^{6,7}$.

The gene mutated in the majority of patients with IMO is called TCIRG1 (or ATP6I or OC116) ${ }^{8}$. The $2,7 \mathrm{kbp}$ transcript codes for the $\alpha 3$ subunit of a proton pump used by the osteoclast to acidify the resorption area ${ }^{9}$. As a consequence of the lack of resorption, remodeling of bone is severely hampered, which results in dense and fragile bone ${ }^{10}$. This in turn causes bone marrow (BM) failure followed by anemia and hepatosplenomegaly ${ }^{1,11,12}$.

The only curative treatment for IMO is HSC transplantation, but this form of therapy is associated with high mortality, especially when HLA-identical donors are not available ${ }^{13}$. IMO is thus a candidate disease for development of gene therapy because of its fatal outcome early in life if treatment with HSC transplantation is not possible. Since progression of the disease is rapid it is of importance to initiate the treatment as 
early as possible. Experience from gene therapy studies of other diseases with hematopoietic involvement, such as X-linked SCID and chronic granulomatous disease ${ }^{14,15}$, show promising results. Gene therapy of IMO has so far not been tested in animal models or in patients. We have previously demonstrated that a mouse model of IMO, the oc/oc mouse, which has a $1500 \mathrm{bp}$ deletion in the tcirgl gene, can be treated with neonatal BM transplantation ${ }^{16}$. Here we show, both in vitro and in vivo, that the osteopetrotic phenotype in oc/oc mice can be reversed by transplantation of fetal liver derived oc/oc HSCs cells retrovirally transduced to express a non-mutated form of tcirg1. This represents the first report of osteoclast directed gene therapy and a first but significant step towards the development of gene therapy for osteopetrosis. 
Materials and methods

Mice

Two pairs of (C57BL/6J x C3HheB/FeJ) $\mathrm{F} 1 \mathrm{oc} /+$ mice (Ly5.2) were obtained from the Jackson Laboratory (Bar Harbour, ME) and maintained in the conventional animal facility at the Biomedical Centre, University of Lund. The mice had a mixed genotype in their HLA-locus $(\mathrm{b} / \mathrm{k})$, and were bred to obtain homozygotes $(\mathrm{b} / \mathrm{b})$. Transplanted oc/oc mice were fed mashed food due to lack of teeth eruption or dental abnormalities in the mice where teeth erupted. Because of abnormal wearing, teeth had to be kept short by cutting. All experiments were performed according to protocols approved by the local ethical committee. Oc/+ mice appeared normal and were phenotypically indistinguishable from wild type (WT) littermates.

\section{Genotyping of mice}

Mice were genotyped on the day of birth using DNA extracted from the tip of the tail. PCR was performed using two forward primers, F1 ( A T C G T A A G C T G G T T G T C C A C T ) a n d F (GATCATGGGCTCTATGGTTCCG), and one reverse primer, R1 (GCTCATTCCATGGGATGTGAATC). Forward primer F3 binds to the sequence deleted in tcirgl in oc/oc mice. For identification of the genotypes the PCR products were loaded onto a $1 \%$ agarose gel. Oc/oc mice were identified by a single band of $306 \mathrm{bp}, \mathrm{oc} /+$ mice by one band of $306 \mathrm{bp}$ and one band of $482 \mathrm{bp}$, and WT mice by a single band of $482 \mathrm{bp}$ (Fig 1D). 
Retroviral vectors and producer cell lines

cDNA for the murine tcirgl gene was amplified from a murine BM cDNA library (generated from a C57/BL6 mouse) using the forward primer TTTCGAAGCTGGAGTGAGCTGCACT and reverse primer CCTGGGCCAGCCAGCTCTTTAT in a first round and forward primer AGCCGAAGTCGACCGAAGAGTCCGCCGGCCACCATGGGCTCTATGTTCCG

$\mathrm{G}$ (for insertions of new restriction sites) and reverse primer TCCCACTTCGGAGAATTCGGGAT in a second round. The amplified cDNA was checked by sequencing. The tcirgl cDNA followed by an internal ribosomal entry site (IRES), GFP cDNA and a woodchuck post-transcriptional regulatory element (wPRE) was cloned into pSF91P ${ }^{17}$, a retroviral vector backbone with a spleen focus-forming virus (SFFV) long terminal repeat (LTR) driving the expression of the transcripts. This vector will be referred to as the tcirg1 vector (Fig 1A). A pSF91P vector expressing GFP preceded by IRES was used as a control vector (Fig 1A). Both vectors were transfected into Phoenix Ampho cells (Nolan lab, Stanford University, USA) and supernatants were harvested for transduction of the stable producer cell line GP+E86 (ecotrophic envelope) followed by selection of high titer clones. The titers were approximately $5 \times 10^{5}$ for the tcirg 1 vector and $1 \times 10^{7}$ for the control vector.

Northern blot on $3 T 3$ cells transduced with the tcirgl vector

$3 \mathrm{~T} 3$ cells were transduced with vector containing medium (VCM) from the tcirg1 stable vector producing cell line and total RNA was extracted using Trizol (Invitrogen, Carlsbad, CA). Untransduced 3T3 cells were used as control cells. Briefly, RNA was separated on an agarose gel and blotted to a membrane. For the 
hybridization, a probe produced by digestion of the tcirg1 vector with FspI (1995 bp band) was used.

\section{Harvest and enrichment of fetal liver hematopoietic cells}

On embryonic day 14.5 pregnant mice were sacrificed by $\mathrm{CO}_{2}$ poisoning, and embryos were removed. Fetal livers (FLs) were dissected out and put into PBS (Invitrogen) supplemented with $2 \%$ FCS (Invitrogen). Single-cell suspensions were prepared by drawing liver cells through a 23 -gauge needle followed by filtering through a 50- $\mu \mathrm{m}$ cell strainer. Individual FLs were genotyped by lysing a cell sample and running the PCR reaction described above for the genotyping of mice. To enrich for stem and progenitor cells Ter119 positive erythroid cells were depleted by incubation with a Ter119 antibody (Pharmingen Becton Dickinson, San Diego, CA) and subsequent incubation with sheep anti-rat antibody-conjugated beads (Dynal Biotech ASA, Oslo, Norway) followed by magnetic separation. Ter119 depleted oc/oc cells were used for gene therapy experiments. For initial transplantation experiments Ter119 depleted WT and +/oc cells were used.

\section{Transduction of fetal liver cells}

24-well suspension culture plates were coated with Retronectin CH-296 (Takara Shuzo, Otsu, Japan), blocked with $2 \%$ BSA in PBS followed by preloading of $125 \mu \mathrm{l}$ VCM. Ter119 depleted FL cells were suspended in VCM supplemented with murine stem cell factor, Flt3-ligand and thrombopoetin, all at $50 \mathrm{ng} / \mathrm{ml}$ (Peprotek, Rocky Hill, $\mathrm{NJ}$ ), and seeded into the wells at a concentration of $6 \times 10^{5}$ cells $/ \mathrm{ml}$. Transduction was repeated after approximately $18 \mathrm{hrs}$ and cells were frozen after a total culture period 
of 24 hrs. Aliquots were kept in culture for an additional 48 hrs and analyzed by flow cytometry to determine the fraction of GFP positive cells.

\section{Transplantations}

One-day-old oc/oc were irradiated with $400 \mathrm{cGy}$ administered from a ${ }^{137} \mathrm{Cs}$ source. Four hours later mice were transplanted i.p with freshly thawed transduced FL cells in $30 \mu 1$ PBS. Oc/oc mice transplanted with oc/oc cells transduced with the tcirg1 vector will be denoted oc/oc+tcirg1 mice.

\section{Engraftment and lineage distribution analysis of peripheral blood}

Peripheral blood (PB) was collected in heparin (LEO Pharma Inc., Thornhill, Ontario, Canada) after tail clipping of mice, put in a 96-well U-bottom plate and mixed with equal volumes of PBS+2\%FCS. Following centrifugation, the supernatant was poured off, erythrocytes lysed with $\mathrm{NH}_{4} \mathrm{Cl}$ and the cells washed twice with PBS+2\%FCS. Subsequently, cells were incubated on ice for 20-30 minutes with APC-conjugated antibodies directed against B220, CD3, Gr-1 and Mac-1 (multi-lineage analysis) (Pharmingen Becton Dickinson). GFP ${ }^{+}$cells were detected in the FL-1 channel. The cells were suspended in $300 \mu \mathrm{PBS}+2 \% \mathrm{FCS}$ followed by addition of $1 \mu \mathrm{g} / \mathrm{ml} 7$ amino-actinomycin D (7-AAD; for detection of non-viable cells, Sigma, St Louis, MO) before analysis using a FACS Calibur Instrument (Becton Dickinson). For engraftment analysis the fraction of $\mathrm{GFP}^{+}$cells was measured.

\section{Isolation and sorting of bone marrow cells from oc/oc+tcirgl and WT mice}

BM from 13 week old oc/oc+tcirg1 and age and gender matched WT mice was isolated as previously described ${ }^{18}$. Mice were killed with a peritoneal injection of a 
lethal dose of Euthesate (8 mg sodium pentobarbital per mouse; Sanofi Santé Animale Benelux B.V., Maassluis, The Netherlands). Femurs and tibiae were removed, cleaned of soft tissue and ground in a mortar with culture medium [ $\alpha$-Minimal Essential Medium (Gibco BRL, Paisley, Scotland)] supplemented with 5\% FCS (HyClone, Logan, UT), $100 \mathrm{U} / \mathrm{ml}$ penicilin, $100 \mu \mathrm{g} / \mathrm{ml}$ streptomycin and $250 \mathrm{ng} / \mathrm{ml}$ amphotericin B (Antibiotic antimyotic solution, Sigma) and heparin (170 IE/ml; Leo Pharmaceutical Products B.V., Weesp, The Netherlands). The cell suspension was aspirated through a 21 gauge needle and filtered over a $100 \mu \mathrm{m}$ pore size Cell Strainer filter (Falcon/Becton Dickinson, Franklin Lakes, NJ). Aliqouts of cells from oc/oc+tcirg 1 mice were sieved through $50 \mu \mathrm{m}$ filters (filcons, Becton Dickinson) before sorting into $\mathrm{GFP}^{+}$and $\mathrm{GFP}^{-}$populations. Depending on the availability of sorters, cells were sorted either on a BD FacsAria (Becton Dickenson) or a MoFlo (Dako, Glostrup, Denmark). Until plating, all cell suspensions were kept on ice.

\section{In vitro osteoclastogenesis}

$\mathrm{BM}$ isolates and sorted cells were plated in 96 wells flat bottom tissue culture treated plates (Costar, Cambridge, MA) at a density of $1 \times 10^{5}$ or $4 \times 10^{5}$ cells per well in $150 \mu 1$ culture medium containing $30 \mathrm{ng} / \mathrm{ml}$ recombinant murine M-CSF (R\&D systems, Mineapolis, MI) with $20 \mathrm{ng} / \mathrm{ml}$ recombinant murine RANKL (RANKL-TEC, R\&D systems). In addition, cells were seeded on $650 \mu \mathrm{m}$ thick bovine cortical bone slices. Culture media were replaced every 3 days. At the end of the culture period, cells were fixed in PBS buffered $4 \%$ formaldehyde and stained for tartrate resistent acid phosphatase (TRACP) activity using the leukocyte acid phosphatase kit (Sigma). Nuclei were stained with diamidino-2phenylindole dihydrochloride (DAPI). 
In vitro bone resorption

For bone resorption assays, the bone slices were cleaned from the cells by incubating the slices in $0.25 \mathrm{M} \mathrm{NH}_{4} \mathrm{OH}$. The slices were washed in distilled water, incubated in a water saturated alum $\left(\mathrm{KAl}\left(\mathrm{SO}_{4}\right)_{2} \cdot 12 \mathrm{H}_{2} \mathrm{O}\right)$ solution, washed in distilled water and stained with Coomassie Brilliant blue. The areas of individual resorption pits were measured using Image-Pro Plus software (MediaCybernetics, Silver Spring, MD).

\section{Quantitative PCR}

RNA was isolated using the RNeasy Mini Kit (Qiagen, Hilden, Germany) according to the manufacturer's instructions. The RNA concentration was measured with the NanoDrop (Nanodrop technologies, Wilmington, USA). 100 - 150 ng RNA was used in the reverse transcriptase reaction which was performed according to the MBI Fermentas cDNA synthesis kit (Vilnius, Lithuania), using both the Oligo(dT)18 and the $\mathrm{D}(\mathrm{N}) 6$ primers. Quantitative real time PCR was performed using SybrGreen I on a LightCycler (Roche Diagnostics GmbH, Mannheim, Germany) for measurement of tcirg1 and hypoxanthine phosphoribosyltransferase (HPRT) as a reference. Two $\mu 1$ of cDNA $(0,5 \mathrm{ng} / \mu \mathrm{l})$ was used in a $10 \mu \mathrm{l}$ final volume reaction containing $1 \mathrm{U}$ Platinum Taq DNA polymerase (Invitrogen, Paisley, UK), 1xbuffer (provided with the enzyme) $0.8 \mathrm{mM}$ dNTP, $3 \mathrm{mM} \mathrm{MgCl} 2,0.5 \mathrm{mg} / \mathrm{ml} \mathrm{BSA} \mathrm{(Sigma} \mathrm{Aldrich),} 5 \%$ DMSO (Sigma Aldrich), $0.5 \mu \mathrm{M}$ forward primer, $0.5 \mu \mathrm{M}$ reverse primer, and 1:20 000 dilution of SybrGreen I. Tcirgl forward primer was GATCATGGGCTCTATGTTCCG, $t$ irg 1 reverse primer ACCTGCCCGCTGCACTTCTT, hprt forward primer was CACAGGACTAGAACACCTGC and hprt reverse primer was GCTGGTGAAAA GGACCTC. 
For provirus copy number determination DNA was isolated from transduced oc/oc fetal liver cells and quantitative PCR was performed as described above using the following primers: Tcirg 1 forward primer GATCATGGGCTCTATGTTCCG and reverse primer GTCTCTGAACTCCACGAG and actin forward primer CAC CACAGCTGAGAGGGAAA and reverse primer GTCAGGCAGCTCATA GCTCTT.

Analysis was done with LightCycler Software version 5.32. Tcirgl PCR values were normalized to the corresponding hprt and actin values.

$X$-ray analysis

Prior to X-ray analysis transplanted or control mice were sacrificed with $\mathrm{CO}_{2}$. The $\mathrm{X}$ ray examination was performed at the University Hospital in Lund.

\section{Histology}

Femurs were dissected from the mice and following fixation in PBS containing $4 \%$ formaldehyde, the bone was put in Parengy decalcification solution $(0.15 \%$ chromethrioxid, $4.3 \%$ nitric acid and $30 \%$ ethanol) (Bie \& Berntsen A-S, Copenhagen, Denmark) for 48 hours. After paraffin embedding, the femurs were subsequently sectioned and stained with Erlish eosin for microscopic examination.

\section{Statistical analysis}

Mean values and standard deviations (SD) were computed in Excel (Microsoft, Redmond, WA). Student's t-test was used for comparisons. Levels of significant difference shown as $*(\mathrm{p}<0.05)$ and $* *(\mathrm{p}<0.005)$. 
Results

Ter119 depleted fetal liver HSCs can be efficiently transduced with retroviral vectors.

We have previously shown that oc/oc mice can be cured by neonatal transplantation of normal BM cells ${ }^{16}$. For gene therapy experiments another source of HSCs had to be used because of the severe reduction of cells in the BM of oc/oc mice. During late embryogenesis the FL serves as the major site for hematopoiesis, and as there are no differences in FL cell number between oc/oc and WT mice (data not shown), these cells were targeted.

FL cells were enriched for HSCs by depletion of erythroid cells using a Ter119 antibody. The average yield of cells after this procedure was $1.9 \times 10^{6}$ cells per FL (range $0.75-2.7 \times 10^{6}$ ). Subsequently cells were transduced in a short 24-hour transduction protocol with either the bicistronic retroviral vector expressing tcirgl and GFP or the GFP control vector. In vitro transduction efficiency was above $90 \%$ with the GFP vector (Table 1) as determined by FACS and somewhat lower with the tcirg1 vector, 42-85 \% (Table 2). The provirus copy number in transduced cells was determined by quantitative PCR and found to be in the range of 3.5-5.5 copies per GFP positive cell when the transduction efficiency was 81\% (SD 3\%, n=6).

Transplantation of normal fetal liver cells transduced with a GFP vector cures oc/oc mice from osteopetrosis and gives rise to long-term engraftment.

To determine if FL HSCs have the same ability to cure oc/oc mice as BM cells, Ter119 depleted WT FL cells transduced with the GFP control vector were transplanted into sub-lethally irradiated 1-day-old oc/oc mice $(n=3$, Table 1$)$. In accordance with our previous study, transplantation of WT hematopoietic cells, although this time from a different source, cured oc/oc mice from osteopetrosis. All 
mice survived past the expected lifespan and exhibited teeth eruption and weight gain in the same manner as seen after the BM transplantation ${ }^{16}$. The percentage of $\mathrm{GFP}^{+}$ cells in PB was analyzed 3, 8 and 18 weeks after transplantation and transduced cells of all hematopoietic lineages were identified (data not shown), indicating the presence of transduced HSCs in the mice.

Neonatal transplantation of oc/oc FL cells transduced with the tcirg1 vector results in long-term survival of oc/oc mice.

Next we determined if oc/oc FL cells, genetically modified to express a non-mutated form of tcirg1, and subsequently transplanted to neonatal oc/oc mice, could rescue these mice from fatal progression of osteopetrosis. Ter119 depleted oc/oc FL cells were transduced with the tcirg1 vector (Fig 1). Transduced cells were transplanted i.p. into 1-day-old mice irradiated with $400 \mathrm{cGy}$ (Fig 1). Eight out of 15 transplanted mice survived past the normal short life-span of oc/oc mice and these are shown in Table 2, as well as the number of cells injected, in vitro transduction efficiency and the level of GFP positive cells in the peripheral blood at various time points. Transplanted oc/oc mice that did not survive died early, 12-22 days after birth. The cell dose and in vitro transduction efficiency of cells transplanted to these mice was in the same range as for surviving mice. Only three of the animals that died early lived long enough (3 weeks) to be subjected to analysis of frequency of $\mathrm{GFP}^{+}$cells in $\mathrm{PB}$, which was below $15 \%$ in all cases. Transplanted mice increased in weight but remained smaller than both normal mice and irradiated normal mice (data not shown). In contrast to mice transplanted with normal BM or FL cells, teeth eruption in the mice transplanted with gene-modified cells was almost absent or very modest. Oc/oc mice transplanted with oc/oc FL cells transduced with the GFP expressing control vector $\left(n=4,3 \times 10^{6}\right.$ 
cells/mouse, $>90 \%$ transduction efficiency in vitro) all died around after 13-15 days showing that the transplantation procedure per se did not prolong the lifespan of treated oc/oc mice.

Multi-lineage engraftment of gene modified cells in transplanted oc/oc mice and reversal of peripheral blood B-cell deficiency.

Reconstitution in oc/oc+tcirg1 mice was analyzed by flow cytometry of PB at different time-points after transplantation. All surviving mice had sustained reconstitution of $\mathrm{GFP}^{+}$cells as shown in Table 2, and also exhibited multi-lineage GFP-marking (Fig 2 A), suggesting transduction of hematopoietic stem or early progenitor cells. It has previously been shown that oc/oc mice have a block in B lymphopoiesis, probably due to reduced production of IL-7 in the BM microenvironment, and as a result a reduced number of B-cells in PB ${ }^{19,20}$. In order investigate if the peripheral B-cell deficiency was corrected, multi-lineage analysis of PB was performed at 8 and 12-18 weeks post transplant (Fig 2 A). We previously showed that PB lineage distribution was normalized in oc/oc mice transplanted with normal $\mathrm{BM}$ cells ${ }^{16}$. In the present study we can see a significant rise in the mature $\mathrm{B} 220^{+}$B-cell population in oc/oc+tcirg1 mice $12-18$ weeks post transplant as compared to untreated oc/oc mice and the level is not significantly different from WT controls (Fig 2 B).

Bone marrow cells harvested from transplanted oc/oc+tcirgl mice form bone resorbing osteoclasts in vitro.

To investigate if transduced oc/oc FL cells can differentiate into bone resorbing osteoclasts we harvested BM cells from oc/oc+tcirg1 mice and cultured these in vitro 
with M-CSF and RANKL. Both green fluorescent and non-fluorescent multinucleated cells formed from oc/oc+tcirg1 BM after 6 days of culture, whereas only nonfluorescent cells formed from WT BM (Fig 3 A). BM from oc/oc+tcirg1 mice was also sorted into populations of $\mathrm{GFP}^{+}$and $\mathrm{GFP}^{-}$cells before in vitro culture. The number of osteoclasts (TRACP positive cells with $\geq 3$ nuclei per cell) that formed was similar between genotypes (WT and oc/oc+tcirg1) and the two sorted cell populations from the oc/oc+tcirg1 mice (Fig $3 \mathrm{~B}$ ). The distribution of the various categories of multinucleated cells (3-5, 6-10 and more than 10 nuclei per cell) was similar between these four groups (data not shown).

We further assessed the in vitro bone resorbing activity of osteoclasts derived from BM cells from WT mice and oc/oc+tcirg1 mice after 6 and 14 days of culture on bovine bone slices. After 6 days, only WT cells exhibited signs of bone resorption (Fig 4), whereas after 14 days of culture also the oc/oc+tcirg1 cells were able to resorb bone, albeit at a lower level than WT cells. When oc/oc+tcirg1 BM was sorted into $\mathrm{GFP}^{+}$and $\mathrm{GFP}^{-}$cell populations and cultured on bone slices, only $\mathrm{GFP}^{+}$cells exhibited bone resorbing activity but not to any greater degree than non-sorted oc/oc+tcirg1 cells. $\mathrm{GFP}^{-}$cells from oc/oc+tcirg1 BM exhibited no signs of bone resorption even after 14 days of culture on bone slices, while osteoclasts derived from oc/oc mice transplanted with normal FL cells transduced with the GFP vector exhibited the same level of resorption as WT cells (data not shown). This indicates that the stem cell source, transduction and transplantation procedure as such did not reduce the resorption capacity of osteoclast-like cells formed.

The level of expression of tcirgl in ocloc+tcirgl osteoclasts is reduced as compared to WT cells. 
Quantitative RT-PCR was performed on cDNA from freshly harvested BM cells (0 days) and in vitro differentiated osteoclasts (6 days) from WT and oc/oc+tcirg1 mice to determine the level of tcirgl expression (Fig 5). In addition, the vector producing cell lines GP+E86 (control vector) and GP+E86 tcirg1 (tcirg1 vector) were used as a comparison of vector expression.

In WT cells, tcirgl was up-regulated as the cells differentiated towards osteoclast like cells, which is in line with previous observations ${ }^{21}$. In freshly harvested BM cells from oc/oc+tcirg1 mice expression from the LTR-driven vector insert was high. However, during the in vitro differentiation towards the osteoclast lineage, the expression of tcirg1 from the vector decreased. Tcirg1 expression could not be detected in the GFP negative cells sorted from oc/oc+tcirg1 mice (data not shown).

$X$-ray examination and histology show formation of bone marrow space

In order to examine the skeletal phenotype mice were analyzed with X-ray and histology. The first analysis was performed 8 weeks post-transplantation. At this time partial remodelling of the bone was observed as detected by X-ray analysis and histology (Fig 6). Analysis 18 weeks after transplantation showed that the bone structure and bone marrow was almost normalized (Fig 6). Transduction of oc/oc HSCs with the tcirg1 vector can thus restore the bone resorbing activity in vivo to a sufficient degree to reverse the osteopetrotic process in oc/oc mice, even though bone resorption in vitro was limited. 


\section{Discussion}

The field of osteoclast biology has developed rapidly during recent years, in part due to knowledge gained from genetic and molecular examination of several mouse models of osteopetrosis, both spontaneous and induced ${ }^{22}$. This condition involves an increase in bone mass, which leads to a number of symptoms of variable severity depending on the mechanism underlying the disease. Osteopetrosis may be caused by defects in osteoclast development or by a reduction in osteoclast function. In terms of correlation between the osteopetrotic mouse models and human forms of these diseases, only mutations affecting osteoclast function have so far been identified as having human counterparts ${ }^{22,23}$. The most severe form of osteopetrosis in humans is IMO. Mutations in three genes, giving rise to three separate single gene disorders all recessive in trait, have so far been shown to cause $\mathrm{IMO}^{2-4,6}$. The most common gene involved is called TCIRG1, the product of which functions as a subunit of a proton pump used by the osteoclast to acidify the space between the cell and underlying bone. The homologue tcirgl is spontaneously mutated in the oc/oc mouse leading to progressive osteopetrosis and death 3-4 weeks after birth. A previous report concluded that the oc/oc mouse was resistant to treatment with BM transplantation ${ }^{24}$. This observation was somewhat puzzling considering that osteoclasts develop from HSCs and that the human form of disease can be treated with HSC transplantation. However, recently, we were able to show that the oc/oc mouse is indeed curable by neonatal transplantation of $\mathrm{BM}$ cells if these, in contrasts to the previous study, are enriched for H2-matched stem cells ${ }^{16}$.

HSCs have been one of the primary targets for gene therapy since these are a readily accessible cell source that can be harvested from patients and re-infused after ex vivo manipulation. However, despite the fact that the osteoclast is a part of the 
hematopoietic system, no gene therapy attempts targeting osteoclasts or their precursors have, to our knowledge, been performed previously.

Here we demonstrate for the first time that osteopetrosis can be reversed by transplantation of gene modified HSCs leading to long-time survival of oc/oc mice suffering from an otherwise rapidly and lethally progressive form of this disease. Eight out of 15 treated oc/oc animals survived past the normal lifespan of oc/oc mice and exhibited signs of osteoclast activity both in vivo and in vitro. In our previously published transplantation study we concluded that all mice with at least 15-20\% of WT donor cells in the PB survived long term while lower levels of engraftment gave variable results. The results in the present study seem to follow the same pattern as all mice with $15 \%$ of GFP positive cells or more in the blood lived past the expected 3-4 week life span of untreated mice. The level of GFP positive cells in peripheral blood was maintained or increased slightly during the life span of the mice and there was also evidence of multi-lineage engraftment indicating stem cell transduction. However, secondary transplants would have to be performed to formally prove stem cell gene transfer.

The overall response rate in the present study (8/15) is approximately the same as seen in a similar murine ex vivo gene therapy study of ADA-SCID ${ }^{25}$. In the present study we choose to deliver the cells by i.p. injection as this resulted in a high survival rate in our previous transplantation study ${ }^{16}$. It is possible, though, that iv injection of gene modified cells would improve the results shown here and maybe also lower the cell dose required.

When osteoclasts were generated and cultured in vitro from BM of oc/oc+tcirg1 mice, there was no sign of bone resorption during a standard culture period of 6 days. Increasing the culture period from 6 to 14 days resulted in bone resorption, however 
to a significantly lower level than mediated by WT cells (about $10 \%$ of normal). Thus partial restoration of osteoclast activity, as determined in vitro, was achieved but not normalization. Theoretically, reasons for this could be impaired development of osteoclasts from transduced cells, a low fraction of corrected cells or a low level of expression of the transgene in the transduced cells. Previously it has been shown that the resorption capacity of osteoclasts is correlated to the number of nuclei in the cells ${ }^{26}$. The in vitro cultures in the present study demonstrate that multinucleated cells are formed to the same extent from oc/oc+tcirg1 cells as from WT cells. Also the number of nuclei per osteoclast did not differ between oc/oc+tcirg1 and WT cells indicating that the differentiation of transduced HSCs into osteoclasts was not impaired in this respect. Even when BM cells from oc/oc+tcirg1 mice were sorted for GFP positive cells to enrich for cells expressing tcirgl and subsequently cultured on bone slices, the level of in vitro bone resorption remained low. This indicates that the low level of resorption cannot be explained by a low fraction of gene corrected cells either. Quantitative PCR, on the other hand, revealed that the expression of tcirgl in osteoclasts derived after in vitro culture of $\mathrm{GFP}^{+}$cells from oc/oc+tcirg1 mice was lower than in osteoclasts that differentiated from WT cells. It is possible that the level of the transgene in the individual osteoclasts is one factor that limits the bone resorbing capacity of the gene corrected cells. Down-regulation of expression from retroviral vectors has previously been described as osteoclast progenitor cells fuse and differentiate into osteoclast-like cells ${ }^{27}$ and also when human CD34 ${ }^{+}$cells differentiate to dendritic cells ${ }^{28}$. In the latter case down-regulation was observed with a number of different vector constructs indicating a general mechanism affecting retroviral enhancer/promoter sequences. For this first study of gene correction of osteopetrosis we selected a vector where the transgene is driven by the strong SFFV 
promoter which generally leads to high expression in most cell types ${ }^{17}$. However, in the future there will be a need for the development of vectors with osteoclast specific promoters that selectively can lead to a high level of expression in mature osteoclasts and hopefully a higher degree of functional correction.

It is interesting and encouraging that despite the fairly low level of correction of osteoclast function observed in vitro there was an almost complete normalization of the skeletal phenotype as judged by histology and X-ray in mice that survived longterm. This indicates that only a small portion of the bone resorbing capacity of osteoclasts is needed to restore the balance between production and destruction of bone in oc/oc mice over time. In comparison to our previous study where we transplanted neonatal mice with WT BM reversal of the phenotype in the current study was slower, and was not observed until the age of 18 weeks compared with 8 weeks for normal BM cells. Other signs of slow bone resorption were also present. Tooth eruption was almost absent in the mice in the present study, in contrast to mice transplanted with WT cells where teeth erupted, albeit not in a normal manner. Even though transplantation of gene modified cells to oc/oc mice may lead to correction of osteopetrosis and long-term survival, the animals are not cured from all manifestations of the disease even if they are transplanted early after birth. We believe that the signs of slow bone resorption and the fact that not all treated mice survive point to the need to enhance osteoclast function early after transplantation. Ways of achieving this could involve, in addition to improving vectors as already mentioned, differentiating part of the transduced cells towards the osteclastic lineage before transplantation or treating transplanted mice with an osteoclast stimulating cytokine, for example RANK-ligand ${ }^{29}$. 
The only existing curative treatment for patients with IMO is HSC transplantation, which is associated with high mortality, especially if an HLA-identical sibling donor is not available ${ }^{13}$. This in combination with the rapid progression of the disease makes gene therapy an attractive option. A potential problem for gene therapy is to obtain a sufficient amount of BM cells for in vitro transduction since affected children have reduced BM cavities due to reduction of the marrow space. However, as IMO patients have increased levels of stem and progenitor cells circulating in the PB, one option would be to harvest $\mathrm{CD} 34^{+}$cells for in vitro transduction from the $\mathrm{PB}$, possibly without need for any prior mobilization regimen to be given ${ }^{30}$. However, it should be stated that a fairly high number of gene corrected cells was transplanted to each recipient in the current study and the amount of cells needed to correct the disease in humans remains to be investigated.

Just as mice transplanted with normal $\mathrm{BM}^{16}$, oc/oc+tcirg1 mice remain growth retarded. Growth retardation is likely to be sustained also in patients after gene therapy since the experience from HSC transplantation show sustained growth impairment even in patients transplanted early (before 3 months of age) ${ }^{13}$. Only transplantation in utero seems to be able to result in normal size of oc/oc mice ${ }^{31}$. However, in the clinical setting, this form of therapy will be an option only in families with a known history of the disease when a prenatal diagnosis is made and it is also technically more complicated to perform.

In conclusion we have demonstrated that the osteopetrosis characteristic for oc/oc mice can be reversed by neonatal transplantation of gene modified hematopoietic stem and progenitor cells. This can lead to long-term survival of treated mice even though only a portion of transplanted cells express the transgene and to a level that is lower than in WT osteoclasts. Our findings represent a first but significant step towards the 
development of gene therapy for osteopetrosis. However, further development of osteoclast specific vectors and optimized transplantation procedures are needed in order to move these findings closer to the clinic. 
Acknowledgements

We thank Lena Persson-Feld and her staff in the animal facility for taking care of the mice and Marie-Louise Olsson and Per Danielsson at Lund University Hospital for help with X-ray analysis. We thank Christopher Baum for the pSF91P vector and Elena Comandasu, Tom o'Toole and Angele Kelder for expert assistance in FACSanalysis and sorting of cells from transplanted mice.

M.J. designed and performed research, collected, analyzed and interpreted data and wrote first draft of manuscript; T.J.dV., T.S and V.E. designed and performed research, collected, analyzed and interpreted data, and wrote parts of the manuscript; A.C.M.B helped in designing, performing and analyzing the qPCR. M.E performed histology analysis; A.F and S.K helped initiate the study and contributed to writing the manuscript; JR initiated and designed the study, analyzed and interpreted data and wrote final version of manuscript. 


\section{References}

1. Fasth A, Porras O. Human malignant osteopetrosis: pathophysiology, management and the role of bone marrow transplantation. Pediatr Transplant. 1999;3 Suppl 1:102-107

2. Kornak U, Schulz A, Friedrich W, Uhlhaas S, Kremens B, Voit T, Hasan C, Bode U, Jentsch TJ, Kubisch C. Mutations in the a3 subunit of the vacuolar H(+)ATPase cause infantile malignant osteopetrosis. Hum Mol Genet. 2000;9:2059-2063

3. Frattini A, Orchard PJ, Sobacchi C, Giliani S, Abinun M, Mattsson JP, Keeling DJ, Andersson AK, Wallbrandt P, Zecca L, Notarangelo LD, Vezzoni P, Villa A. Defects in TCIRG1 subunit of the vacuolar proton pump are responsible for a subset of human autosomal recessive osteopetrosis. Nat Genet. 2000;25:343-346

4. Kornak U, Kasper D, Bosl MR, Kaiser E, Schweizer M, Schulz A, Friedrich W, Delling G, Jentsch TJ. Loss of the ClC-7 chloride channel leads to osteopetrosis in mice and man. Cell. 2001;104:205-215

5. Frattini A, Pangrazio A, Susani L, Sobacchi C, Mirolo M, Abinun M, Andolina M, Flanagan A, Horwitz EM, Mihci E, Notarangelo LD, Ramenghi U, Teti A, Van Hove J, Vujic D, Young T, Albertini A, Orchard PJ, Vezzoni P, Villa A. Chloride channel $\mathrm{ClCN} 7$ mutations are responsible for severe recessive, dominant, and intermediate osteopetrosis. J Bone Miner Res. 2003;18:1740-1747

6. Chalhoub N, Benachenhou N, Rajapurohitam V, Pata M, Ferron M, Frattini A, Villa A, Vacher J. Grey-lethal mutation induces severe malignant autosomal recessive osteopetrosis in mouse and human. Nat Med. 2003;9:399-406

7. Rajapurohitam V, Chalhoub N, Benachenhou N, Neff L, Baron R, Vacher J. The mouse osteopetrotic grey-lethal mutation induces a defect in osteoclast maturation/function. Bone. 2001;28:513-523 
8. Sobacchi C, Frattini A, Orchard P, Porras O, Tezcan I, Andolina M, BabulHirji R, Baric I, Canham N, Chitayat D, Dupuis-Girod S, Ellis I, Etzioni A, Fasth A, Fisher A, Gerritsen B, Gulino V, Horwitz E, Klamroth V, Lanino E, Mirolo M, Musio A, Matthijs G, Nonomaya S, Notarangelo LD, Ochs HD, Superti Furga A, Valiaho J, van Hove JL, Vihinen M, Vujic D, Vezzoni P, Villa A. The mutational spectrum of human malignant autosomal recessive osteopetrosis. Hum Mol Genet. 2001;10:17671773

9. Li YP, Chen W, Stashenko P. Molecular cloning and characterization of a putative novel human osteoclast-specific $116-\mathrm{kDa}$ vacuolar proton pump subunit. Biochem Biophys Res Commun. 1996;218:813-821

10. Wilson CJ, Vellodi A. Autosomal recessive osteopetrosis: diagnosis, management, and outcome. Arch Dis Child. 2000;83:449-452

11. Coccia PF, Krivit W, Cervenka J, Clawson C, Kersey JH, Kim TH, Nesbit ME, Ramsay NK, Warkentin PI, Teitelbaum SL, Kahn AJ, Brown DM. Successful bone-marrow transplantation for infantile malignant osteopetrosis. N Engl J Med. $1980 ; 302: 701-708$

12. Gerritsen EJ, Vossen JM, van Loo IH, Hermans J, Helfrich MH, Griscelli C, Fischer A. Autosomal recessive osteopetrosis: variability of findings at diagnosis and during the natural course. Pediatrics. 1994;93:247-253

13. Driessen GJ, Gerritsen EJ, Fischer A, Fasth A, Hop WC, Veys P, Porta F, Cant A, Steward CG, Vossen JM, Uckan D, Friedrich W. Long-term outcome of haematopoietic stem cell transplantation in autosomal recessive osteopetrosis: an EBMT report. Bone Marrow Transplant. 2003;32:657-663

14. Cavazzana-Calvo M, Hacein-Bey S, de Saint Basile G, Gross F, Yvon E, Nusbaum P, Selz F, Hue C, Certain S, Casanova JL, Bousso P, Deist FL, Fischer A. 
Gene therapy of human severe combined immunodeficiency (SCID)-X1 disease. Science. 2000;288:669-672

15. Ott MG, Schmidt M, Schwarzwaelder K, Stein S, Siler U, Koehl U, Glimm H, Kuhlcke K, Schilz A, Kunkel H, Naundorf S, Brinkmann A, Deichmann A, Fischer M, Ball C, Pilz I, Dunbar C, Du Y, Jenkins NA, Copeland NG, Luthi U, Hassan M, Thrasher AJ, Hoelzer D, von Kalle C, Seger R, Grez M. Correction of Xlinked chronic granulomatous disease by gene therapy, augmented by insertional activation of MDS1-EVI1, PRDM16 or SETBP1. Nat Med. 2006;12:401-409

16. Johansson M, Jansson L, Ehinger M, Fasth A, Karlsson S, Richter J. Neonatal hematopoietic stem cell transplantation cures oc/oc mice from osteopetrosis. Exp Hematol. 2006;34:242-249

17. Schambach A, Wodrich H, Hildinger M, Bohne J, Krausslich HG, Baum C. Context dependence of different modules for posttranscriptional enhancement of gene expression from retroviral vectors. Mol Ther. 2000;2:435-445

18. de Vries TJ, Schoenmaker T, Beertsen W, van der Neut R, Everts V. Effect of CD44 deficiency on in vitro and in vivo osteoclast formation. J Cell Biochem. 2005;94:954-966

19. Blin-Wakkach C, Wakkach A, Sexton PM, Rochet N, Carle GF. Hematological defects in the oc/oc mouse, a model of infantile malignant osteopetrosis. Leukemia. 2004;18:1505-1511

20. Blin-Wakkach C, Wakkach A, Quincey D, Carle GF. Interleukin-7 partially rescues B-lymphopoiesis in osteopetrotic oc/oc mice through the engagement of B220(+)CD11b(+) progenitors. Exp Hematol. 2006;34:851-859 
21. Toyomura T, Oka T, Yamaguchi C, Wada Y, Futai M. Three subunit a isoforms of mouse vacuolar $\mathrm{H}(+)$-ATPase. Preferential expression of the a3 isoform during osteoclast differentiation. J Biol Chem. 2000;275:8760-8765

22. Van Wesenbeeck L, Van Hul W. Lessons from osteopetrotic mutations in animals: impact on our current understanding of osteoclast biology. Crit Rev Eukaryot Gene Expr. 2005;15:133-162

23. Tolar J, Teitelbaum SL, Orchard PJ. Osteopetrosis. N Engl J Med. 2004;351:2839-2849

24. Seifert MF, Marks SC, Jr. Congenitally osteosclerotic (oc/oc) mice are resistant to cure by transplantation of bone marrow or spleen cells from normal littermates. Tissue Cell. 1987;19:29-37

25. Mortellaro A, Hernandez RJ, Guerrini MM, Carlucci F, Tabucchi A, Ponzoni M, Sanvito F, Doglioni C, Di Serio C, Biasco L, Follenzi A, Naldini L, Bordignon C, Roncarolo MG, Aiuti A. Ex vivo gene therapy with lentiviral vectors rescues adenosine deaminase (ADA)-deficient mice and corrects their immune and metabolic defects. Blood. 2006;108:2979-2988

26. Boissy P, Saltel F, Bouniol C, Jurdic P, Machuca-Gayet I. Transcriptional activity of nuclei in multinucleated osteoclasts and its modulation by calcitonin. Endocrinology. 2002;143:1913-1921

27. Selski DJ, Clohisy DR. A customized retroviral vector confers marker gene expression in osteoclast lineage cells. J Cell Biochem. 2006;97:641-650

28. Lindemann C, Schilz AJ, Emons B, Baum C, Low R, Fauser AA, Kuehlcke K, Eckert HG. Down-regulation of retroviral transgene expression during differentiation of progenitor-derived dendritic cells. Exp Hematol. 2002;30:150-157 
29. Kollet O, Dar A, Shivtiel S, Kalinkovich A, Lapid K, Sztainberg Y, Tesio M, Samstein RM, Goichberg P, Spiegel A, Elson A, Lapidot T. Osteoclasts degrade endosteal components and promote mobilization of hematopoietic progenitor cells. Nat Med. 2006;12:657-664

30. Steward CG, Blair A, Moppett J, Clarke E, Virgo P, Lankester A, Burger SR, Sauer MG, Flanagan AM, Pamphilon DH, Orchard PJ. High peripheral blood progenitor cell counts enable autologous backup before stem cell transplantation for malignant infantile osteopetrosis. Biol Blood Marrow Transplant. 2005;11:115-121

31. Frattini A, Blair HC, Sacco MG, Cerisoli F, Faggioli F, Cato EM, Pangrazio A, Musio A, Rucci F, Sobacchi C, Sharrow AC, Kalla SE, Bruzzone MG, Colombo R, Magli MC, Vezzoni P, Villa A. Rescue of ATPa3-deficient murine malignant osteopetrosis by hematopoietic stem cell transplantation in utero. Proc Natl Acad Sci U S A. 2005;102:14629-14634 
Figure legends

Figure 1. Vector design and experimental set-up. (A) Design of retroviral vectors. The backbone in both vectors contains SFFV LTRs and a wPRE. The upper vector contains murine tcirgl cDNA followed by an IRES and GFP and is denoted the tcirg1 vector. The lower vector control vector contains an IRES sequence followed by GFP. (B) Northern blot showing mRNA expression of tcirgl in the transduced cell line 3T3. (C) FL harvest, Ter119 depletion, in vitro transduction and analysis. (D) Genotyping, irradiation and transplantation of oc/oc mice.

Figure 2. Multilineage reconstitution of transduced cells in oc/oc+tcirg1 mice and reversal of peripheral blood B-cell deficiency in oc/oc+tcirg1 mice. A) The peripheral blood of an 18 week old oc/oc+tcirg1 mice was analysed for expression of GFP and lineage markers. Percentage positive cells as indicated in the graph. B) The percentage of B220 positive cells in the peripheral blood of oc/oc, oc/oc+tcirg1 and WT mice was analysed at the time points indicated. The mean value and SD is shown $(n=4-6$ mice per group)

Figure 3. Cells harvested from oc/oc+tcirg1 mice can be in vitro differentiated to osteoclast-like cells in normal numbers. BM cells from oc/oc+tcirg1 mice were cultured for 6 days with M-CSF and RANK-L after which generation of osteoclastlike cells was analysed. A. Both $\mathrm{GFP}^{+}$(I, II) and $\mathrm{GFP}^{-}$(III, IV) osteoclast-like cells were formed. (I, III): Green fluorescent; (II, IV): blue fluorescent (DAPI) image. Bar $=100 \mu \mathrm{m}$. Microscope Leica DM IL, camera Leica DFC320 and acquisition software Leica IM500. B. Osteoclasts were cultured on bone slices from BM cells obtained from WT or oc/oc+tcirg1 mice. oc/oc+tcirg1 cells were also FACS-sorted into GFP ${ }^{+}$ 
and GFP populations. After culture with M-CSF and RANKL for 6 days the number of multinucleated ( $\geq 3$ nuclei) cells was enumerated. Mean \pm SD is shown.

Figure 4. In vitro differentiated cells from oc/oc+tcirg1 mice are capable of bone resorption. Osteoclasts were cultured from BM cells harvested from WT or oc/oc+tcirg1 mice. In addition, oc/oc+tcirg1 cells were FACS-sorted into $\mathrm{GFP}^{+}$and GFP' populations. After culture with M-CSF and RANKL for 6 or 14 days on cortical bone slices cells were removed and resorption pits were visualized. A. Examples of bone resorption pits formed by WT, oc/oc+tcirg1, oc/oc+tcirg1 $\left(\mathrm{GFP}^{+}\right)$and oc/oc tcirg1 (GFP') derived osteoclasts after 6 (upper panel) and 14 days (lower panel). Bar $=100 \mu \mathrm{m}$. Microscope and camera model, see fig 3. B. Percentage of bone surface resorbed after 6 (empty bars) or 14 (solid bars) days of culture. Mean \pm SD of a triplicate plating is shown.

Figure 5. Expression of WT tcirg1 is up-regulated during osteoclast differentiation while vector mediated expression is down-regulated. Quantitative RT-PCR was performed on cDNA from freshly isolated BM cells (0 days) harvested from WT and oc/oc+tcirg1 mice $\left(\mathrm{GFP}^{+}\right.$cells) and after 6 days of culture with M-CSF and RANKL to determine the level of tcirg1 expression. Tcirg1 PCR values were normalized against the corresponding HPRT value giving a relative intensity value for comparison of different samples. Expression levels are compared to the tcirg1 vector $\mathrm{GP}+\mathrm{E} 86$ producer cell line (striped bar) and the GFP vector GP+E86 producer cell line (grey bar). 
Figure 6. Reversal of osteopetrotic phenotype in oc/oc+tcirg1 mice as demonstrated by X-ray and histology. The left column (A-D) shows X-ray images and the right column (E-H) shows the corresponding histology images (femur). Threeweek-old untreated control mouse $(\mathrm{A}+\mathrm{E})$, a 3-week-old untreated oc/oc mouse $(\mathrm{B}+\mathrm{F})$, 8-week-old oc/oc+tcirg1 mouse $(\mathrm{C}+\mathrm{G})$, and 18-week-old oc/oc+tcirg1 mouse (D+H). Note that bone marrow volume increases at the expense of bone volume in oc/oc+tcirg1 compared to untreated oc/oc mouse. Olympus BX45 microscope and Nikon Prixera PRO 150 ES was used for the histology images. 
Table 1. Successful long-term reconstitution of GFP vector transduced normal FL cells in oc/oc mice.

\begin{tabular}{cccccc}
\hline & & & \multicolumn{3}{c}{$\% \mathrm{GFP}^{+}$cells in PB } \\
\cline { 4 - 6 } Mouse & \% GFP in vitro & No. of cells & $\mathbf{3 w}$ & $\mathbf{8 w}$ & $\mathbf{1 8 w}$ \\
\hline 1 & 90 & $1,8 \times 10^{6}$ & 10 & 54 & 61 \\
2 & 90 & $1.8 \times 10^{6}$ & 64 & 60 & 78 \\
3 & 95 & $2,0 \times 10^{6}$ & 26 & 78 & 57 \\
\hline
\end{tabular}


Table 2. Successful transplantation of tcirg1 vector transduced oc/oc FL cells to oc/oc mice and long-term survival of 8 out of 15 treated mice.

\begin{tabular}{|c|c|c|c|c|c|c|}
\hline \multirow[b]{2}{*}{ Mouse } & \multirow{2}{*}{$\begin{array}{l}\text { \% GFP } \\
\text { in vitro }\end{array}$} & \multirow{2}{*}{$\begin{array}{c}\text { No. of } \\
\text { cells }\end{array}$} & \multicolumn{3}{|c|}{$\% \mathrm{GFP}^{+}$cells in $\mathrm{PB}$} & \multirow[b]{2}{*}{ Outcome } \\
\hline & & & $3 w$ & 6-8w & $12-18 w$ & \\
\hline 1 & 67 & $1,0 \times 10^{6}$ & 21 & 23 & $\mathrm{NA}$ & $\mathrm{S}$ at $8 \mathrm{w}$ \\
\hline 2 & 85 & $1,3 \times 10^{6}$ & 35 & 25 & NA & $\mathrm{S}$ at $8 \mathrm{w}$ \\
\hline 3 & 42 & $2,7 \times 10^{6}$ & 13 & 20 & 37 & $\mathrm{~S}$ at $23 \mathrm{w}$ \\
\hline 4 & 55 & $3,5 \times 10^{6}$ & 14 & 49 & 54 & $\mathrm{~S}$ at $18 \mathrm{w}$ \\
\hline 5 & 55 & $3,5 \times 10^{6}$ & 8 & 4 & 5 & $\mathbf{t}$ at $24 \mathrm{w}$ \\
\hline 6 & 66 & $1,7 \times 10^{6}$ & 26 & 28 & 33 & $\mathrm{~S}$ at $13 \mathrm{w}$ \\
\hline 7 & 66 & $1,7 \times 10^{6}$ & 25 & 22 & 20 & $\mathrm{~S}$ at $20 \mathrm{w}$ \\
\hline 8 & 70 & $1,6 \times 10^{6}$ & 29 & 32 & 26 & $\mathrm{SA}$ at $12 \mathrm{w}$ \\
\hline
\end{tabular}

Sacrificed for in vitro osteoclastogenesis, X-ray and histology (S),

Died (t), Not applicable (NA), Still alive (SA) 
Figure 1

SFFV tcirg1 IRES GFP wPRE SFFV tcirg1 vector

SFFV $\quad$ RES GFP WPRE SFFV control vector
1)

C

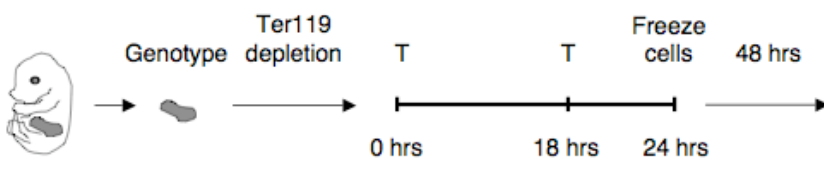

FL

In vitro transduction (T)

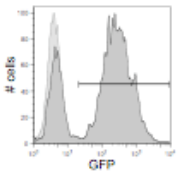

FACS of cell sample
E14.5 FL

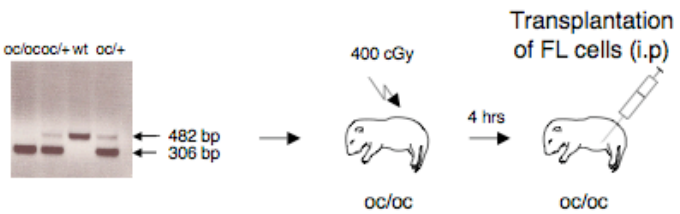

d 0

d 1 
Figure 2

A
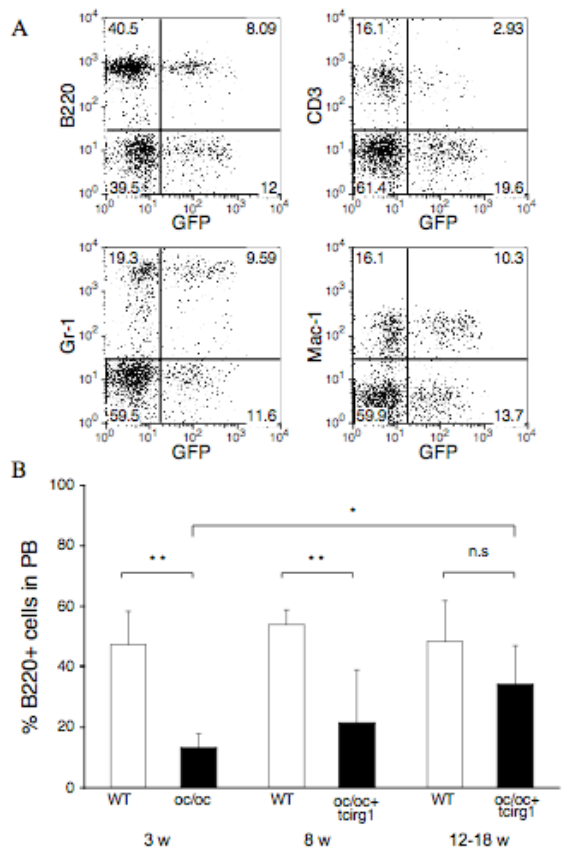
Figure 3
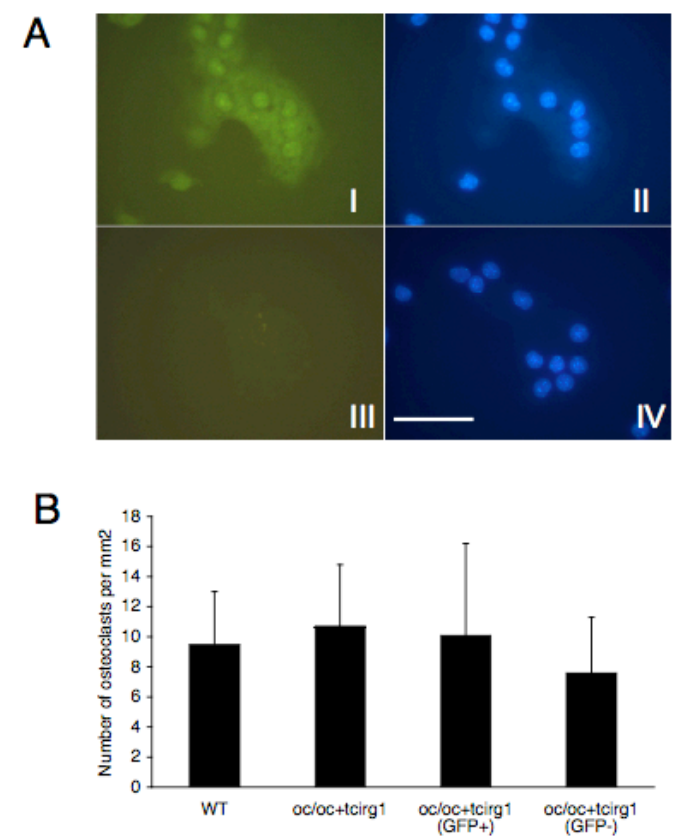
Figure 4

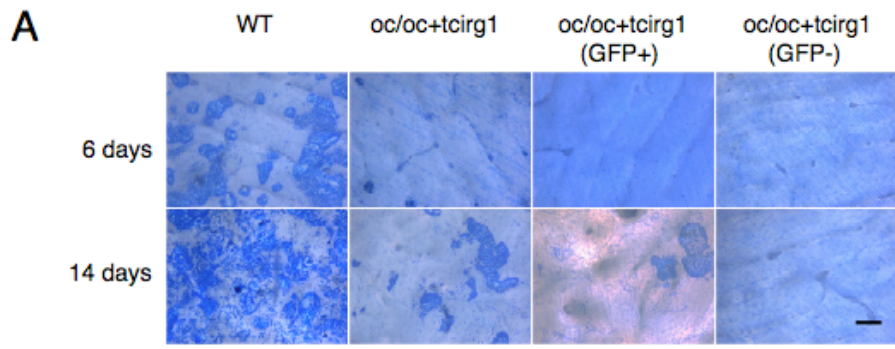

B

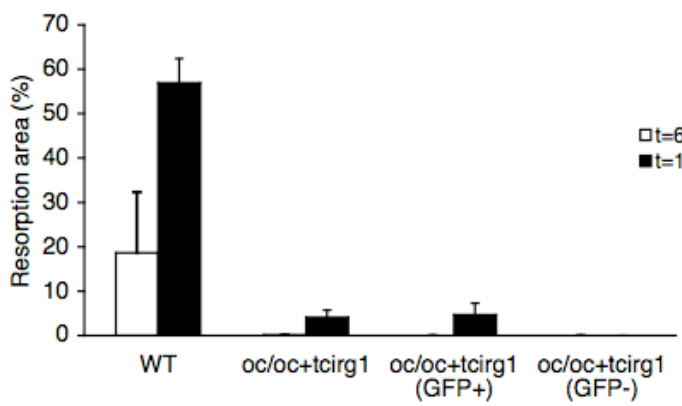


Figure 5

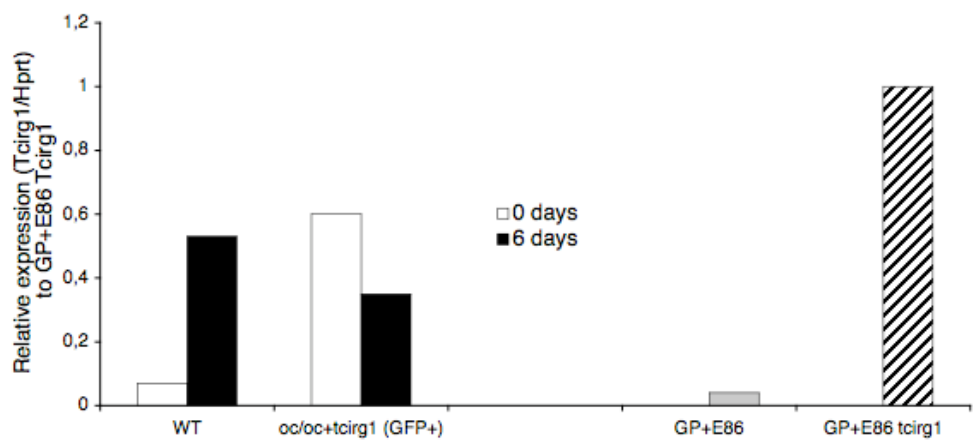


Figure 6

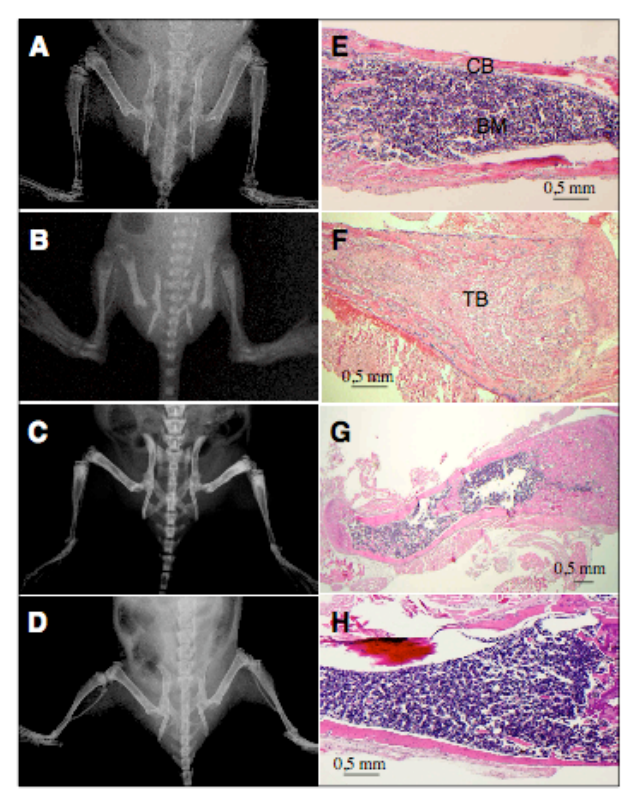

